\title{
El reto del Perú frente a los Mega Acuerdos TPP y TTIP.
}

\author{
Breve revisión a algunos aportes teóricos recientes
}

Mg. Econ. y Ms. Sc. Katia MEJÍA SÁENZ ${ }^{29}$

\section{RESUMEN:}

En el presente artículo, se hace un breve análisis de las cambiantes condiciones del mercado mundial, donde nuestro país deberá tomar el reto de enfrentar los nuevos mega acuerdos comerciales que se están negociando y los cuales tienen como objetivo reactivar los mercados productivos, aumentar el intercambio comercial, mejorar el Ingreso y el Consumo de los países involucrados. La diversidad y asimetría de las economías que participan en estas negociaciones impregnará mayor dinamismo, así como mayores distancias entre los países en cuanto a sus metas de desarrollo, innovación e incremento de su oferta agregada. Es por esto que nuestro país debería estar preparándose para este gran reto. Me permito hacer una revisión de las investigaciones más importantes de la teoría económica que pueda permitir una propuesta de política económica.

\begin{abstract}
:
In this article, we make a brief tour of the changing conditions of the world market, where our country should take the challenge of facing the new "mega trade agreements" being negotiated which aim is to reactivate the productive markets, increase exchange trade, improve the income and consumption of the countries involved. The diversity and asymmetry of the economies participating in these negotiations will create more dynamic, as well as greater distances between countries in terms of their development goals, innovation and aggregate supply. That is why our country should be preparing for this challenge. Let me make a review of the most recent researches of economic theory in order to allow the proposal of an economic policy.
\end{abstract}

La creciente y dinámica integración e interdependencia de los diversos mercados financieros internacionales, la expansión del comercio internacional, la creación

29 Instituto de Investigaciones de la FACEE. Cualquier consulta a kms230793@gmail.com 
de bloques económicos regionales, los acuerdos de libre comercio y cooperación económica, la apertura al exterior de las grandes economías emergentes y el ascenso de la economía asiática en el escenario mundial, así como las continuas innovaciones tecnológicas y organizativas de gestión que desde mediados de los años setenta en el siglo XX y con mayor fuerza en el siglo XXI, vienen canalizando el tránsito desde la economía de la información a la sociedad del conocimiento, son algunos de los fenómenos asentados en la raíz de la globalización de la economía y por ende, de los mercados.

La crisis financiera del año 2008 es sin duda una de las más globales y ha afectado al Centro Industrializado, a diferencia de las anteriores, ya que casi por lo general se originaban en la Periferia Económica. El nuevo siglo nos presenta un escenario económico mundial diferente, con cambios en los actores, los mercados, la dirección de los flujos y la composición de los mismos.

La explosión de la burbuja inmobiliaria en el año 2008, trajo entre otras consecuencias, la contracción del crédito, empeorando las condiciones mundiales del sistema financiero, pues todos los países del mundo requieren (en diferente intensidad) crédito para poder mantener la Inversión, la actividad productiva, por ende, el comercio internacional, el empleo y el crecimiento.

La Globalización y la crisis financieras internacionales han generado cambios inevitables en los escenarios de los mercados mundiales y cambios en los paradigmas que han signado la teoría y la práctica, los modelos y las tendencias estudiados y difundidos desde las escuelas de negocios a nivel mundial. El mundo industrializado aún no logra restablecer el equilibrio macroeconómico en sus centros y los planes de rescate, así como combinaciones de instrumentos monetarios y fiscales, no logran revertir el efecto de la crisis del 2008. La Empresa ha buscado caminos para poder subsistir primero y luego intentar el crecimiento, a través de nuevos canales.

La interdependencia de los mercados hace que la contracción de la demanda externa desde las economías industrializadas, impacte negativamente en el comercio exterior de los socios de contrapartida, es decir, de los Países en Desarrollo y Países Emergentes, por lo que las consecuencias de una crisis financiera internacional tiene efectos globales, impactando en prácticamente todos los indicadores. La apertura económica y comercial, los procesos de integración económica, la globalización y la dinámica de la tecnología hacen que la interdependencia económica sea cada vez más intensa, por lo que todos los países (Gobiernos, Empresas, Academia) deben avocarse al desarrollo e implementación de estrategias que permitan no solo revertir los efectos de los desequilibrios macroeconómicos, pero buscar recomponer el crecimiento ${ }^{30}$. 
El ratio de los intercambios comerciales internacionales respecto del PIB mundial pasó el $13 \%$ en la década de los 70' a un promedio del 30\% hacia el inicio de la presente década. Esta es una de muchas medidas para poder sopesar el proceso de globalización de los mercados. La globalización es un fenómeno macroeconómico visible y tangible, pero se realiza prioritariamente a nivel de corporaciones, empresas multinacionales, grandes y medianas empresas, donde la pequeña empresa aùn no logra marcar presencia.

Los enfoques de la teoría económica se han ido ajustando en función a los nuevos y cambiantes escenarios de la economía mundial. Un ejemplo de ello es el Nuevo Enfoque de la Teoría del Comercio que nos ha mostrado y demostrado los beneficios del sano intercambio; otros que han variado han sido: el Modelo Hecksher Ohlin, la Teoría del Ciclo del Producto, las Economías de Escala en mercados imperfectamente competitivos, la hipótesis de la Similitud de las Preferencias, Competencia Monopolística y Comercio, el Modelo Estándar. Todos estos enfoques nos han explicado las bases, los procesos, los factores intervinientes, las variables y los efectos a lo largo de más de medio siglo, a través de los drásticos cambios en los escenarios y condiciones de los mercados internacionales.

La fuerza de la Innovación y la tecnología han tenido y tienen un impactante efecto en la economía mundial, en el comercio, en las negociaciones y las políticas industriales y comerciales que toda nación debe diseñar y desarrollar en función a sus propias características y necesidades.

Para Helpman y Krugman (1985) las diferencias en los costos comparativos relacionados con la depreciación factor o la tecnología eran necesarias y suficientes para el surgimiento de un comercio internacional, a pesar de que tales diferencias se mantuvieran consistentes con la existencia de intercambios cruzados de bienes similares. Este enfoque ha sido la espina dorsal de los trabajos de economía internacional aplicada, como en el caso del modelo de gravedad(o gravitacional) del intercambio comercial. En el centro medular de este enfoque ubicamos a la empresa representativa que ostenta el monopolio sobre la diversidad de bienes producidos por ésta, pero, sometida a la competencia de otras variedades (de otras firmas), en ausencia de barreras de entrada a los mercados.

Jan Tinbergen (1962) se inspiró en la ley de gravedad enunciada por Newton para describir la dinámica de los intercambios bilaterales. En física, dos cuerpos se atraen en relación directa a su masa y en relación inversa a la distancia que separa sus centros de gravedad. Según la ecuación de gravedad ( gravitacional) del comercio internacional establecida por Tinbergen, el volumen de intercambios (X) que realizan dos países A y B entre ellos, es por un lado, proporcional a su producto Bruto Interno ( PIB) y por otro, inversamente proporcional a la distancia (d) que los separa. 
Los PIB en realidad corresponden a M1 y M2, que a su vez representan la Masa, en la ecuación base. $\boldsymbol{d}$ es la distancia que separa los objetos, en este caso, a los países intervinientes, $\boldsymbol{G}$ es una constante gravitacional y X (intercambio) corresponde a $\boldsymbol{F}$ o la fuerza de atracción

$$
\mathrm{X}=\mathrm{G} \times \frac{\mathrm{PIB}_{A}^{\alpha} \times \mathrm{PIB}_{B}^{\beta}}{\mathrm{d}^{\gamma}}
$$

Dicho de otra forma, mientras mayor sea el tamaño de las economías socias intervinientes en el intercambio, mayor será el volumen (quantum) de intercambio entre ambos. Mientras más alejados uno del otro (los países), menos importantes resultarán sus intercambios.

Los investigadores Anne -Célia Disdier y Keith Head ${ }^{31}$ compilaron en el año 2006, un total de 1467 estimaciones de esta ecuación, a partir de 103 artículos y encontraron que un incremento en un 10\% de la Distancia, se traduce en promedio, en una reducción de $9 \%$ del intercambio comercial bilateral. Estos coeficientes se mostraron estables en el tiempo y espacio, si bien la ecuación aparece "como una de las regularidades empíricas más estables y robustas en los aportes económicos contemporáneos"32. Deducimos entonces que los acuerdos de comercio mejoran las condiciones de comercio, reduciendo los costos de transporte, los que son considerados en la ecuación como d, por lo que necesariamente el Perú debe participar de estos mega - acuerdos.

La importancia del tamaño de la economía de mercado y su intercambio con el exterior ha sido demostrada ampliamente por Paul Krugman, quien muestra como el volumen del intercambio de un país con el resto del mundo es proporcional a su tamaño y negativamente afectado por las barreras comerciales.

Dado este axioma, todos los países tienen un especial interés en especializarse en una determinada variedad y diversidad de opciones de un bien o producto (o línea de producto) y en la etapa más evolucionada de Internacionalización en una diversidad de servicios, de tal manera que el beneficio del intercambio sea más importante gracias a las economías de escala. Asimismo, buscarán importar otros bienes y servicios con mayores ventajas a fin de reducir el costo de producción en caso de tratarse de commodities e insumos industriales, y de mejorar el bienestar del consumidor.

La ecuación podría ser considerada como la interpretación de la oferta y demanda que influyen en toda operación de comercio, volviéndola especial para cada caso,

31 The Puzzling Persistence of the Distance Effect on Bilateral Trade," Review of Economics and Statistics, 2008.

32 Ibid. 
tanto como son especiales y diferentes todos los mercados y todos los sectores, como son diferentes los productos y los servicios.

Los Acuerdos comerciales juegan entonces un rol cada vez más preponderante, ya no solo debido a la fuerza que impregnan al intercambio, sino por la dinámica que los procesos de Internacionalización están inyectando a todos los mercados. La Liberalización comercial ha entrado en una etapa crucial, cambiante, en parte debido a la explosión de la actual crisis, y en opinión de la suscrita, ha preparado el terreno para una nueva forma de liberalización de los mercados financieros y cambiarios.

En párrafos anteriores se señala que la presente década está caracterizada - en materia de Negociaciones- por la que un comercio abierto más dinámico que busca mayores espacios y zonas geográficas y por ello se celebran Mega acuerdos como el Trans Pacific Partnership (TPP) y Transatlantic Trade and Investment Partnership (TTIP).

El TPP es una negociación entre 12 países, que a su vez representan un $36 \%$ de la producción mundial y el $11 \%$ de la población y aproximadamente un tercio del comercio o intercambio a nivel mundial. Perú es parte de estas negociaciones y el reto es bastante alto, pues de firmarse, nuestra economía, en realidad nuestros empresarios, tendrán que competir con sus pares de economías de diversos tamaños y perfiles, a bajos costos y con estándares internacionales laborales, industriales, sanitarios y técnicos, descontada por supuesto, la calidad.

El TTIP es el Tratado Transatlántico de comercio e Inversiones que celebrarán EE.UU y la Unión Europea, y representa el 46\% de la producción mundial y aproximadamente el 28\% del comercio. Este Tratado es exclusivo de los dos mayores grupos comerciales, dejando de lado a la República Popular China, quien a su vez es miembro de APEC más no participa de las negociaciones TPP.

En ambos casos, las barreras de ingreso a los mercados son muy diversas y aún bastante sólidas, tanto en términos arancelarios, como en medidas para-arancelarias. La crisis financiera ha sido el argumento principal para que algunas medidas de protección hayan sido reactivadas en las economías del centro. El tema de los derechos de propiedad intelectual, el comercio de servicios, las protecciones laborales y políticas más favorables al medio ambiente forman parte de las rondas previas de discusión, a fin de lograr una mayor coherencia en las futuras relaciones económicas internacionales de estos socios.

Estamos por lo tanto, ante la posibilidad de ingresar a una etapa de acuerdos plurilaterales que puedan ser normados a nivel supranacional, de tal manera que las asimetrías existentes entre los futuros socios permitan un intercambio algo más equilibrado y algo más equitativo. 


\section{CONCLUSIONES}

1. La teoría nos demuestra que es necesario acercar distancias y ajustar costos a fin de lograr mayores beneficios.

2. El reto es muy grande, los acuerdos por celebrarse en el futuro inmediato son muy complejos, los mercados en competencia son muy agresivos, diversificados y competitivos.

3. La realidad y la casuística nos presentan barreras existentes dentro de nuestro propio mercado, y que competen al Gobierno que debe implementar el ordenamiento, el respeto al estado de derecho, la supervisión y la regulación, a fin que la empresas puedan acceder a condiciones que les permitan competir en el mercado mundial que hoy más que nunca se presenta como dinámico, complejo, recesado y cambiante.

\section{REFERENCIAS BIBLIOGRÁFICAS}

1. DiSDIER ANNE-CÉliA ET KEITH HEAD (2008). The Puzzling Persistence of the Distance Effect on Bilateral Trade," Review of Economics and Statistics.

2. ELHANAN HELPMAN (1987). Imperfect Competition and International Trade: Evidence from Fourteen Industrial Countries en: JOURNAL OF THE JAPANESE AND INTERNATIONAL ECONOMIES I,62431.

3. KENNETH A. REINERT, Gravity Models, en: http://press.princeton.edu/ chapters/reinert/19article_reinert gravity.pdf

4. KRUGMAN PAUL (1999). Economía internacional, 3 ed. Mc Graw Hill.

5. MEJÍA SÁENZ KATIA (2014). Las crisis Internacionales, Texto para la FACEE, URP.

6. RIALP. A. y RIALP. J. (2005). Las formas actuales de penetración y desarrollo de los mercados internacionales, carcaterización, marcos conceptuales y evidencia empírica en el caso español, claves de la economía mundial, ICEX, Madrid.

7. VILLARREAL LARRINAGA OSKAR (2014). la Internacionalización de la empresa: el modelo de las 10 estrategias, UAB Barcelona.

\section{WEBGRAFÍA}

1. https://www.wto.org/indexsp.htm

2. http://www.imf.org/external/spanish/

3. http://www.icex.es/icex/es/index.html

4. http://www.mincetur.gob.pe/newweb/

5. http://www.uab.cat/

6. http://www.cato.org/

7. http://campus-montreal.ca/nouvelles/ 